\title{
Some New Integral Inequalities via Variant of Pompeiu's Mean Value Theorem
}

\author{
Mehmet ZeKI SARIKAYA
}

ABstract. The main of this paper is to establish an inequality providing some better bounds for integral mean by using a mean value theorem. Our results generalize the results of Ahmad et. al in [8].

\section{INTRODUCTION}

The inequality of Ostrowski [7] gives us an estimate for the deviation of the values of a smooth function from its mean value. More precisely, if $f:[a, b] \rightarrow \mathbb{R}$ is a differentiable function with bounded derivative, then

$$
\left|f(x)-\frac{1}{b-a} \int_{a}^{b} f(t) \mathrm{d} t\right| \leq\left[\frac{1}{4}+\frac{\left(x-\frac{a+b}{2}\right)^{2}}{(b-a)^{2}}\right](b-a)\left\|f^{\prime}\right\|_{\infty}
$$

for every $x \in[a, b]$. Moreover the constant $1 / 4$ is the best possible.

For a differentiable function $f:[a, b] \rightarrow \mathbb{R}, a \cdot b>0$, Dragomir has proved in [2], using Pompeiu's mean value theorem [5], the following Ostrowski type inequality:

$$
\left|\frac{a+b}{2} \cdot \frac{f(x)}{x}-\frac{1}{b-a} \int_{a}^{b} f(t) \mathrm{d} t\right| \leq D(x)\left\|f-\ell f^{\prime}\right\|_{\infty}
$$

where $\ell(t)=t, t \in[a, b]$, and

$$
D(x)=\frac{(b-a)}{|x|}\left[\frac{1}{4}+\frac{\left(x-\frac{a+b}{2}\right)^{2}}{(b-a)^{2}}\right] .
$$

In [4], Pecaric and Ungar proved a general estimate with the $p$-norm, $1<$ $p<\infty$, which for $p=1$ will give the Dragomir [2] result.

2000 Mathematics Subject Classification. 26D10, 26D15, $41 \mathrm{~A} 55$.

Key words and phrases. Ostrowski inequality, $p$-norm, mean value theorem. 
In [8], for a twice differentiable function $f:[a, b] \rightarrow \mathbb{R}, a \cdot b>0$ Farooq et. al gave the following integral inequality:

$$
\begin{aligned}
& \left|\frac{a+b}{2}\left(\frac{2 f(x)}{3 x}-\frac{f^{\prime}(x)}{2}\right)+\frac{1}{3}\left(\frac{b f(b)-a f(a)}{b-a}\right)-\frac{1}{b-a} \int_{a}^{b} f(t) \mathrm{d} t\right| \\
\leq & \frac{(b-a)}{3|x|}\left[\frac{1}{4}+\frac{\left(x-\frac{a+b}{2}\right)^{2}}{(b-a)^{2}}\right]\left\|2 f-2 \ell f^{\prime}+\ell^{2} f^{\prime \prime}\right\|_{\infty}
\end{aligned}
$$

where $\ell(u)=u, u \in[a, b]$.

The interested reader is also referred to ([2]-[4], [6], [8], [9]) for integral inequalities by using Pompeiu's mean value theorem.

In this paper, we establish an general form with the $p$-norm, $1 \leq p \leq \infty$, which will give the Ahmad et. al result for $p=\infty$. Our results generalize the results of Ahmad et. al in [8].

\section{Main Results}

Before stating the main results, we will give the following lemma proved by Pecaric and Ungar in [4]:

Lemma 2.1. For $\frac{1}{p}+\frac{1}{q}=1,1 \leq p, q \leq \infty$, and $0<a \leq x \leq b$, denote

$$
A(x, q):=\left(\int_{a}^{x}\left(\int_{t}^{x} \frac{t^{q} \mathrm{~d} u}{u^{2 q}}\right) \mathrm{d} t\right)^{\frac{1}{q}}+\left(\int_{x}^{b}\left(\int_{x}^{t} \frac{t^{q} \mathrm{~d} u}{u^{2 q}}\right) \mathrm{d} t\right)^{\frac{1}{q}}
$$

where for $p=1$, i.e. $q=\infty$, the integrals are to be interpreted as the $\infty$ norms, i.e. as maxima of the function $(u, t) \mapsto \frac{1}{u^{2}}$ on the corresponding domains of integration. Then,

$$
\begin{aligned}
A(x, q)= & \left(\frac{a^{2-q}-x^{2-q}}{(1-2 q)(2-q)}+\frac{x^{2-q}-a^{1+q} x^{1-2 q}}{(1-2 q)(1+q)}\right)^{\frac{1}{q}} \\
& +\left(\frac{b^{2-q}-x^{2-q}}{(1-2 q)(2-q)}+\frac{x^{2-q}-b^{1+q} x^{1-2 q}}{(1-2 q)(1+q)}\right)^{\frac{1}{q}},
\end{aligned}
$$

for $1<p, q<\infty, p, q \neq 2$;

$$
\begin{gathered}
A(x, 2)=\frac{1}{3}\left[\left(\ln \left(\frac{x}{a}\right)^{3}+\frac{a^{3}}{x^{3}}-1\right)^{\frac{1}{2}}+\left(\ln \left(\frac{x}{b}\right)^{3}+\frac{b^{3}}{x^{3}}-1\right)^{\frac{1}{2}}\right]=\lim _{q \rightarrow 2} A(x, q) ; \\
A(x, \infty)=\frac{a^{2}+b^{2}}{2 x}+x-a-b=\lim _{q \rightarrow \infty} A(x, q) ; \\
A(x, 1)=\frac{1}{a}+\frac{b}{x^{2}}=\lim _{q \rightarrow 1} A(x, q) .
\end{gathered}
$$

To prove our theorems, we need the following lemma: 
Lemma 2.2. Let $f:[a, b] \rightarrow \mathbb{R}$ be continuous function on $[a, b]$ and twice order differentiable function on $(a, b)$ with $0<a<b$. Then for any $t, x \in$ $[a, b]$, we have

$$
\begin{aligned}
t f(x)- & x f(t)+x t \frac{f^{\prime}(t)-f^{\prime}(x)}{2}= \\
& \frac{x t}{2} \int_{x}^{t}\left[2 f(u)-2 u f^{\prime}(u)+u^{2} f^{\prime \prime}(u)\right] \frac{1}{u^{2}} \mathrm{~d} u .
\end{aligned}
$$

Proof. Define $\Psi:\left[\frac{1}{b}, \frac{1}{a}\right] \rightarrow \mathbb{R}$ by $\Psi(t):=t^{2} f\left(\frac{1}{t}\right)$. The function $\Psi$ is continuously differentiable on $\left(\frac{1}{b}, \frac{1}{a}\right)$, and for all $x_{1}, x_{2} \in\left[\frac{1}{b}, \frac{1}{a}\right]$, we get

$$
\begin{aligned}
& \Psi^{\prime}\left(x_{1}\right)-\Psi^{\prime}\left(x_{2}\right)=\int_{x_{2}}^{x_{1}} \Psi^{\prime \prime}(t) \mathrm{d} t \\
= & \int_{x_{2}}^{x_{1}}\left[2 f\left(\frac{1}{t}\right)-\frac{2}{t} f^{\prime}\left(\frac{1}{t}\right)+\frac{1}{t^{2}} f^{\prime \prime}\left(\frac{1}{t}\right)\right] \mathrm{d} t .
\end{aligned}
$$

Using the change of the variable in last integrals with $u=\frac{1}{t}$, we get

$$
\Psi^{\prime}\left(x_{1}\right)-\Psi^{\prime}\left(x_{2}\right)=-\int_{\frac{1}{x_{2}}}^{\frac{1}{x_{1}}}\left[2 f(u)-2 u f^{\prime}(u)+u^{2} f^{\prime \prime}(u)\right] \frac{1}{u^{2}} \mathrm{~d} u .
$$

Denote $x_{1}=\frac{1}{x}$ and $x_{2}=\frac{1}{t}$. Then for all $x, t \in[a, b]$ from (3), we have

$$
\begin{aligned}
& \frac{2}{x} f(x)-f^{\prime}(x)-\frac{2}{t} f(t)+f^{\prime}(t)= \\
& \quad \int_{x}^{t}\left[2 f(u)-2 u f^{\prime}(u)+u^{2} f^{\prime}(u)\right] \frac{1}{u^{2}} \mathrm{~d} u
\end{aligned}
$$

which gives (2) and completes the proof.

Theorem 2.1. Let $f:[a, b] \rightarrow \mathbb{R}$ be continuous function on $[a, b]$ and twice order differentiable function on $(a, b)$ with $0<a<b$. Then for $\frac{1}{p}+\frac{1}{q}=1$, with $1 \leq p, q \leq \infty$, and all $x \in[a, b]$, we have

$$
\begin{aligned}
& \left|\frac{a+b}{2}\left(\frac{2 f(x)}{3 x}-\frac{f^{\prime}(x)}{3}\right)+\frac{1}{3}\left(\frac{b f(b)-a f(a)}{b-a}\right)-\frac{1}{b-a} \int_{a}^{b} f(t) \mathrm{d} t\right| \\
\leq & \frac{(b-a)^{\frac{1}{p}-1}}{3}\left\|2 f-2 \ell f^{\prime}+\ell^{2} f^{\prime \prime}\right\|_{p} A(x, q)
\end{aligned}
$$

where $\ell(u)=u, u \in[a, b]$. 
Proof. From Lemma 2.2, we have

$$
\begin{aligned}
& t f(x)-x f(t)+x t \frac{f^{\prime}(t)-f^{\prime}(x)}{2} \\
= & \frac{x t}{2} \int_{x}^{t}\left[2 f(u)-2 u f^{\prime}(u)+u^{2} f^{\prime}(u)\right] \frac{1}{u^{2}} \mathrm{~d} u .
\end{aligned}
$$

Integrating with respect to $t$ on $[a, b]$ and dividing by $\frac{3 x}{2}$, we get

$$
\begin{aligned}
& \frac{\left(b^{2}-a^{2}\right)}{2}\left(\frac{2 f(x)}{3 x}-\frac{f^{\prime}(x)}{3}\right)+\frac{b f(b)-a f(a)}{3}-\int_{a}^{b} f(t) \mathrm{d} t \\
= & \int_{a}^{b} \frac{t}{3}\left(\int_{x}^{t}\left[2 f(u)-2 u f^{\prime}(u)+u^{2} f^{\prime}(u)\right] \frac{1}{u^{2}} d u\right) \mathrm{d} t
\end{aligned}
$$

and therefore

(6)

$$
\begin{aligned}
& \left|\frac{\left(b^{2}-a^{2}\right)}{2}\left(\frac{2 f(x)}{3 x}-\frac{f^{\prime}(x)}{3}\right)+\frac{b f(b)-a f(a)}{3}-\int_{a}^{b} f(t) \mathrm{d} t\right| \\
\leq & \int_{a}^{b}\left(\left|\int_{x}^{t}\right|\left[2 f(u)-2 u f^{\prime}(u)+u^{2} f^{\prime \prime}(u)\right] \frac{t}{3 u^{2}}|d u|\right) \mathrm{d} t \\
= & \int_{a}^{x}\left(\left|\int_{x}^{t}\right|\left[2 f(u)-2 u f^{\prime}(u)+u^{2} f^{\prime \prime}(u)\right] \frac{t}{3 u^{2}}|d u|\right) \mathrm{d} t \\
& +\int_{x}^{b}\left(\left|\int_{x}^{t}\right|\left[2 f(u)-2 u f^{\prime}(u)+u^{2} f^{\prime \prime}(u)\right] \frac{t}{3 u^{2}}|d u|\right) \mathrm{d} t .
\end{aligned}
$$

Firstly, we consider the case $1<p, q<\infty$. By using Hölder's inequality, the sum in the last line of (6) can be written

$$
\begin{gathered}
\left(\int_{a}^{x}\left(\int_{t}^{x}\left|2 f(u)-2 u f^{\prime}(u)+u^{2} f^{\prime}(u)\right|^{p} \mathrm{~d} u\right) \mathrm{d} t\right)^{\frac{1}{p}}\left(\int_{a}^{x}\left(\int_{t}^{x} \frac{t^{q} \mathrm{~d} u}{u^{2 q} 3^{q}}\right) \mathrm{d} t\right)^{\frac{1}{q}} \\
+\left(\int_{x}^{b}\left(\int_{x}^{t}\left|2 f(u)-2 u f^{\prime}(u)+u^{2} f^{\prime \prime}(u)\right|^{p} \mathrm{~d} u\right) \mathrm{d} t\right)^{\frac{1}{p}}\left(\int_{x}^{b}\left(\int_{x}^{t} \frac{t^{q} \mathrm{~d} u}{u^{2 q} 3^{q}}\right) \mathrm{d} t\right)^{\frac{1}{q}}
\end{gathered}
$$




$$
\begin{aligned}
& \leq \frac{1}{3}\left(\int_{a}^{b}\left(\int_{a}^{b}\left|2 f(u)-2 u f^{\prime}(u)+u^{2} f^{\prime \prime}(u)\right|^{p} \mathrm{~d} u\right) \mathrm{d} t\right)^{\frac{1}{p}} \\
& \quad \times\left[\left(\int_{a}^{x}\left(\int_{t}^{x} \frac{t^{q} \mathrm{~d} u}{u^{2 q}}\right) \mathrm{d} t\right)^{\frac{1}{q}}+\left(\int_{x}^{b}\left(\int_{x}^{t} \frac{t^{q} \mathrm{~d} u}{u^{2 q}}\right) \mathrm{d} t\right)^{\frac{1}{q}}\right] .
\end{aligned}
$$

The first factor in (7) is equal with

$$
\begin{aligned}
& \left(\int_{a}^{b}\left(\int_{a}^{b}\left|2 f(u)-2 u f^{\prime}(u)+u^{2} f^{\prime \prime}(u)\right|^{p} \mathrm{~d} u\right) \mathrm{d} t\right)^{\frac{1}{p}} \\
= & (b-a)^{\frac{1}{p}}\left\|2 f-2 \ell f^{\prime}+\ell^{2} f^{\prime \prime}\right\|_{p} .
\end{aligned}
$$

and, by Lemma 2.1, the second factor equals $A(x, q)$. Thus, putting (8) into (6) and dividing $b-a$ gives the required inequality (4).

Theorem 2.2. Let $f:[a, b] \rightarrow \mathbb{R}$ be continuous function on $[a, b]$ and twice order differentiable function on $(a, b)$ with $0<a<b$, and let $w:[a, b] \rightarrow \mathbb{R}$ be a nonnegative integrable function. Then for $\frac{1}{p}+\frac{1}{q}=1$ with $1 \leq p, q \leq \infty$ any $t, x \in[a, b]$, we have

(9)

$$
\begin{gathered}
\left.\mid\left(\frac{2 f(x)-x f^{\prime}(x)}{2 x}\right) \int_{a}^{b} t w(t) \mathrm{d} t-\int_{a}^{b} w(t) f(t) \mathrm{d} t+\frac{1}{2} \int_{a}^{b} t w(t) f^{\prime} t\right) \mathrm{d} t \mid \\
\leq(b-a)^{\frac{1}{p}}\left\|2 f-2 \ell f^{\prime}+\ell^{2} f^{\prime \prime}\right\|_{p}\left[\left(\int_{a}^{x} \frac{\left[x^{1-2 q} t^{q}-t^{1-q}\right] w^{q}(t)}{(1-2 q)} \mathrm{d} t\right)^{\frac{1}{q}}\right. \\
\left.+\left(\int_{x}^{b} \frac{\left[t^{1-q}-x^{1-2 q} t^{q}\right] w^{q}(t)}{(1-2 q)} \mathrm{d} t\right)^{\frac{1}{q}}\right]
\end{gathered}
$$

where $\ell(u)=u, u \in[a, b]$.

Proof. Multiplying (5) by $\frac{w(t)}{x}$ and integrating with respect to $t$ on $[a, b]$, we have

$$
\left(\frac{2 f(x)-x f^{\prime}(x)}{2 x}\right) \int_{a}^{b} t w(t) \mathrm{d} t-\int_{a}^{b} w(t) f(t) d t+\frac{1}{2} \int_{a}^{b} t w(t) f^{\prime}(t) \mathrm{d} t
$$




$$
=\frac{1}{2} \int_{a}^{b} t w(t)\left(\int_{x}^{t}\left[2 u f(u)-2 u f^{\prime}(u)+u^{2} f^{\prime \prime}(u)\right] \frac{1}{u^{2}} \mathrm{~d} u\right) \mathrm{d} t
$$

and as in the proof of Theorem 2.1, we get

$$
\begin{aligned}
& \left|\left(\frac{2 f(x)-x f(x)}{2 x}\right) \int_{a}^{b} t w(t) \mathrm{d} t-\int_{a}^{b} w(t) f(t) \mathrm{d} t+\frac{1}{2} \int_{a}^{b} t w(t) f^{\prime}(t) \mathrm{d} t\right| \\
& \leq \frac{1}{2} \int_{a}^{b}\left|\int_{x}^{t}\right| 2 f(u)-2 u f^{\prime}(u)+u^{2} f^{\prime \prime}(u)\left|\frac{t w(t)}{u^{2}} \mathrm{~d} u\right| \mathrm{d} t \\
& =\int_{a}^{x}\left(\int_{t}^{x}\left|2 f(u)-2 u f^{\prime}(u)+u^{2} f^{\prime \prime}(u)\right| \frac{t w(t)}{u^{2}} \mathrm{~d} u\right) \mathrm{d} t \\
& +\int_{x}^{b}\left(\int_{x}^{t}\left|2 f(u)-2 u f^{\prime}(u)+u^{2} f^{\prime \prime}(u)\right| \frac{t w(t)}{u^{2}} \mathrm{~d} u\right) \mathrm{d} t \\
& \leq\left[\int_{a}^{x} \int_{c}^{y}\left(\int_{t}^{x} \int_{s}^{y}\left|2 f(u)-2 u f^{\prime}(u)+u^{2} f^{\prime \prime}(u)\right|^{p} \mathrm{~d} u\right) \mathrm{d} t\right]^{\frac{1}{p}} \\
& {\left[\int_{a}^{x}\left(\int_{t}^{x} \frac{t^{q} w^{q}(t) \mathrm{d} u}{u^{2 q}}\right) \mathrm{d} t\right]^{\frac{1}{q}}} \\
& +\left[\int_{x}^{b}\left(\int_{x}^{t}\left|2 f(u)-2 u f^{\prime}(u)+u^{2} f^{\prime \prime}(u)\right|^{p} \mathrm{~d} u\right) \mathrm{d} t\right]^{\frac{1}{p}} \\
& {\left[\int_{x}^{b}\left(\int_{x}^{t} \frac{t^{q} w^{q}(t) \mathrm{d} u}{u^{2 q}}\right) \mathrm{d} t\right]^{\frac{1}{q}}} \\
& \leq\left[\int_{a}^{b}\left(\int_{a}^{b}\left|2 f(u)-2 u f^{\prime}(u)+u^{2} f^{\prime \prime}(u)\right|^{p} \mathrm{~d} u\right) \mathrm{d} t\right]^{\frac{1}{p}} \\
& \times\left(\left[\int_{a}^{x}\left(\int_{t}^{x} \frac{t^{q} w^{q}(t) \mathrm{d} u}{u^{2 q}}\right) \mathrm{d} t\right]^{\frac{1}{q}}+\left[\int_{x}^{b}\left(\int_{x}^{t} \frac{t^{q} w^{q}(t) \mathrm{d} u}{u^{2 q}}\right) \mathrm{d} t\right]^{\frac{1}{q}}\right)
\end{aligned}
$$

which gives (9). 


\section{REFERENCES}

[1] A.M. Acu, A. Baboş and F.D. Sofonea, The mean value theorems and inequalities of Ostrowski type, Sci. Stud. Res. Ser. Math. Inform. 21 (2011), no. 1, 5-16.

[2] S.S. Dragomir, An inequality of Ostrowski type via Pompeiu's mean value theorem, J. of Inequal. in Pure and Appl. Math., 6(3) (2005), Art. 83.

[3] I. Muntean, Extensions of some mean value theorems, Babes-Bolyai University, Faculty of Mathematics, Research Seminars on Mathematical Analysis, Preprint Nr. 7, 1991, 7-24.

[4] P.P. Pecaric and S. Ungar, On an inequality of Ostrowski type, J. of Inequal. in Pure and Appl. Math., 7(4) (2006), Art. 151.

[5] D. Pompeiu, Sur une proposition analogue au théorème des accroissements finis, Mathematica (Cluj, Romania), 22 (1946), 143-146.

[6] E.C. Popa, An inequality of Ostrowski type via a mean value theorem, General Mathematics Vol. 15, No. 1, 2007, 93-100.

[7] A. Ostrowski, Uber die Absolutabweichung einer differentierbaren Funktionen von ihrem Integralmittelwert,Comment. Math. Helv., 10(1938), 226-227.

[8] F. Ahmad, N.A. Mir and M.Z. Sarikaya, An inequality of Ostrowski type via variant of Pompeiu's mean value theorem,J. Basic. Appl. Sci. Res., 4(4)204-211, 2014.

[9] P.K. Sahoo and T. Riedel, Mean Value Theorems and Functional Equations, World Scientific, Singapore, New Jersey, London, Hong Kong, 2000.

Mehmet Zeki Sarikaya

Department of Mathematics

Faculty of Science And Arts

DÜZCE UNIVERSITY

DÜZCE

TURKEY

E-mail address: sarikayamz@gmail.com 